\title{
Core momentum and particle transport studies in the ASDEX Upgrade tokamak
}

\author{
R. M. McDermott ${ }^{1}$, C. Angioni ${ }^{1}$, R. Dux ${ }^{1}$, E. Fable ${ }^{1}$,

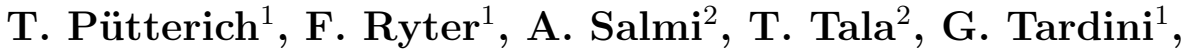 \\ E. Viezzer ${ }^{1}$ and the ASDEX Upgrade Team ${ }^{1}$ \\ ${ }^{1}$ Max Planck Institut für Plasmaphysik, EURATOM Association, D-85748 Garching, \\ Germany \\ ${ }^{2}$ Association EURATOM-Tekes, VTT, P.O. Box 1000, FIN-02044 VTT, Finland \\ E-mail: Rachael.McDermott@ipp.mpg.de
}

\begin{abstract}
Core momentum and particle transport in ASDEX Upgrade (AUG) have been examined in a wide variety of plasma discharges and via several different methods. Experiments were performed in which ECRH power was added to NBI heated H-modes causing the electron and impurity ion density profiles to peak and the core toroidal rotation to flatten. Turbulence calculations of these plasmas show a change in the dominant regime from ITG to TEM due to the ECRH induced changes in the electron and ion temperature profiles. The impurity and electron density behavior can be fully explained by the changes in the turbulent particle transport. Momentum transport analyses demonstrate that in the TEM regime there is a core localized, counter-current directed, residual stress momentum flux of the same order of magnitude as the applied NBI torque. The initial results from momentum modulation experiments performed on AUG confirm that the Prandtl number in AUG NBI heated H-modes is close to 1 and that there exists a significant inward momentum pinch. Lastly, an intrinsic toroidal rotation database has been developed at AUG which can be used to test theoretically predicted dependencies of residual stress momentum fluxes. Initial results show a linear correlation between the gradient of the toroidal rotation and both the electron density gradient scale length and the frequency of the dominant turbulent mode.
\end{abstract}

\section{Introduction}

The realization of the connections between the plasma rotation profile and plasma stability and confinement has made momentum transport an active area of tokamak research. It is commonly accepted that velocity shear can lead to improved confinement through the suppression of turbulence [1, 2, 3, 4, 5] and high rotation is known to be beneficial to stability against a variety of MHD modes including resistive wall modes [6, 7, 8, and neoclassical tearing modes [9, 10]. For these reasons it is desirable to be able to predict and, ultimately, have active control over the rotation behavior in future fusion devices. Similarly, a thorough understanding of electron and impurity particle transport is equally important to the success of commercial fusion energy. In a fusion reactor, it 
will be necessary to maintain a peaked electron density profile, or at least a sufficiently high core electron density, in order to optimize energy production [11, 12]. However, it will also be necessary to remove helium ash from the plasma core and to prevent the build up of high-Z impurities, which can radiate away the plasma energy. These constraints place considerable importance on achieving a fundamental understanding of both momentum and particle transport in fusion plasmas.

In recent years significant progress has been made toward a theoretical understanding of particle transport and, perhaps more importantly, much of the theory has been validated through comparison of predicted and experimentally observed behaviors, see [12, 13, 14, 15, 16] and references therein. Although our theoretical understanding of momentum transport has developed appreciably [17, 18, 19, 20, 21, 22, 23, 24, 25, 26], validation of momentum theory through comparison to experiment is still in its early stages [27, 28, 29, 30, 31, 32, 33, 34, 35]. In particular, our understanding of intrinsic momentum fluxes remains largely empirical [36, 37, 38, 39, 40, 41, 42, 43. and detailed comparison between theoretically predicted residual stress mechanisms and experimental observations of intrinsic rotation remains an open area of research. This is, at least in part, due to the complexity of the experimental observations and the difficulty in designing experiments which can adequately test the available theory. In this work, the authors attempt to merge observations from a wide variety of ASDEX Upgrade (AUG) discharges into a consistent description of core, intrinsic toroidal rotation.

This paper is organized as follows: In section II studies of core particle and momentum transport in H-modes heated with a combination of neutral beam injection (NBI) and electron cyclotron resonance heating (ECRH) are presented. In addition, the initial results from momentum modulation experiments on AUG are described. Section III introduces the first measurements of intrinsic rotation at AUG and presents results from the newly developed intrinsic toroidal rotation database. The key findings and conclusions are summarized in Section IV.

\section{Momentum and particle transport in NBI heated H-modes}

\section{II.1. Description of plasma behavior}

Previous work on AUG examined core particle and momentum transport in low current (600 kA) H-mode discharges heated with a combination of ECRH and NBI power [40, 13. In purely NBI heated discharges the ion and electron temperature profiles are very similar and through linear calculations with the code GS2 [44, 45] it was determined that these plasmas are solidly in the ion temperature gradient (ITG) dominated turbulence regime. However, with ECRH the electron and ion temperatures can be separated leading to $T_{e} / T_{i}$ ratios in the plasma core of order 2-4 and moving the plasma into the trapped electron mode (TEM) dominated regime. In the NBI only cases the plasmas spin up in the co-current direction and form peaked rotation profiles, but with ECRH the rotation decreases significantly leading to flat and even slightly hollow 

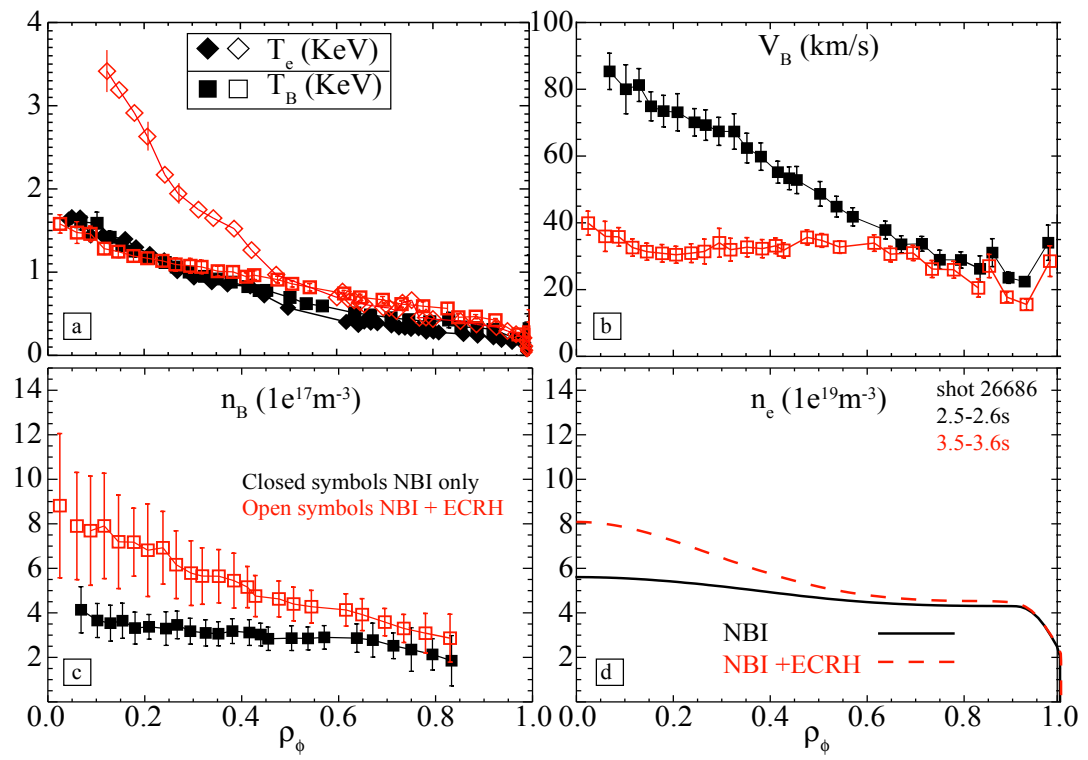

Figure 1. Comparison of ion and electron temperature (a), toroidal rotation (b), and boron and electron density profiles (c and d) in a 2.5MW NBI H-mode with (open/red) and without (full/black) $2 \mathrm{MW}$ of central ECRH power.

profiles. The electron and boron density profiles are also affected. In the NBI only cases they are flat or only moderately peaked, but as the plasmas transition from ITG to TEM they are observed to peak with the largest changes in gradient around mid-radius. An example of these profile behaviors can be found in Fig. 1 for a $0.6 \mathrm{MA}$ H-mode heated with 2.5 MW of NBI. Here, and in the rest of this work, positive (negative) toroidal rotation velocities correspond to co-current (counter-current) rotation.

Recently, these experiments were repeated with significantly improved diagnostic capability and covering a wider range of parameter space. The primary improvements in diagnostic capability are to the core charge exchange recombination spectroscopy (CXRS) system, which was upgraded to increase the level of signal and the number of lines of sight, enabling faster integration times and more detailed radial profiles. These upgrades significantly improve the accuracy of the measured impurity ion temperature and toroidal rotation profiles, see Fig.1 (a) and (b). The new experiments include NBI heated H-mode discharges with plasma currents between 0.6 MA and $1 \mathrm{MA}$ and core plasma densities up to $10^{20} \mathrm{~m}^{-3}$. In all discharges very similar behavior was observed in all of the plasma profiles indicating the robustness of the observed phenomenology. All of the experiments presented in this work (including section III) were conducted in the "forward field" configuration, in which the plasma current is in the counter-clockwise direction and the toroidal field in the clockwise direction as seen from a top down view of the tokamak. In this configuration the neutral beam momentum input is in the co-current direction. 


\section{II.2. Electron and impurity particle transport}

A detailed analysis of the electron and boron particle transport in these plasmas can be found in reference [13. This work shows that the peaking of the electron density with ECRH, despite appearing to contradict previous observations of density pump-out with ECRH, can be fully explained by the theoretically predicted changes in the turbulent particle transport as the plasma transitions from ITG to TEM. Density pump-out is observed in low density L-mode discharges and in high density H-mode discharges [12]. The turbulence induced peaking of the electron density has a maximum in the TEM regime, while the frequency of the turbulent mode is still close to zero [46, 16]. Low density L-mode discharges are typically TEM dominated and are already at or past this maximum. Hence, the addition of ECRH leads to a reduction in the density peaking (pump-out) by driving the turbulence deeper into the TEM regime. High density H-modes, on the other hand, are ITG dominated and the high collisionality makes separating $T_{e}$ and $T_{i}$ difficult. In these plasmas the addition of moderate amounts of ECRH does not significantly alter the turbulence regime, but can mitigate the core density gradient by increasing the core transport and decreasing the Ware pinch [47]. Low and moderate density NBI H-modes, like those presented here, are ITG dominated, but with ECRH power transition toward TEM, causing the observed peaking in the density profiles.

Reference [13] also shows that the boron density behavior is well described by the modeling if the term in the turbulent impurity flux expression proportional to the gradient of the plasma rotation (roto-diffusion) is included in the calculations [48]. In this analysis of the boron transport very good agreement between theory and experiment was obtained for low and high ECRH power cases, but only moderate agreement was obtained for plasmas heated with only NBI power. The modeling was unable to reproduce the degree of hollowness observed in the experimental profiles, see Fig. 12 of reference [13]. Recently, it was found that the optics of the CXRS lines of sight used for the boron density measurements were degraded during the experimental campaign and that the boron density profiles are more peaked than they first appeared. This changes the level of agreement between theory and experiment that was reported in reference [13. The theoretical predictions remain unchanged, it is only the experimental boron density gradients that are shifted to slightly higher values. A comparison of the theoretically predicted and the corrected experimental values of $R / L_{n B}$ (the normalized boron density gradient scale length) as a function of the boron Mach number is shown in Fig. 2(a). Here, one can see that the theory still reproduces the experimental trends quite well. However, the agreement is somewhat altered with respect to the original analysis; in the NBI only cases (blue squares) it is improved as the profiles are no longer quite so hollow, while in the high ECRH power cases it is somewhat reduced.

In Fig. 2(b) the same comparison is shown except that here the roto-diffusion contribution to the turbulent particle flux has been excluded. The reduced agreement illustrates the importance of the roto-diffusion term to correctly predicting the impurity 

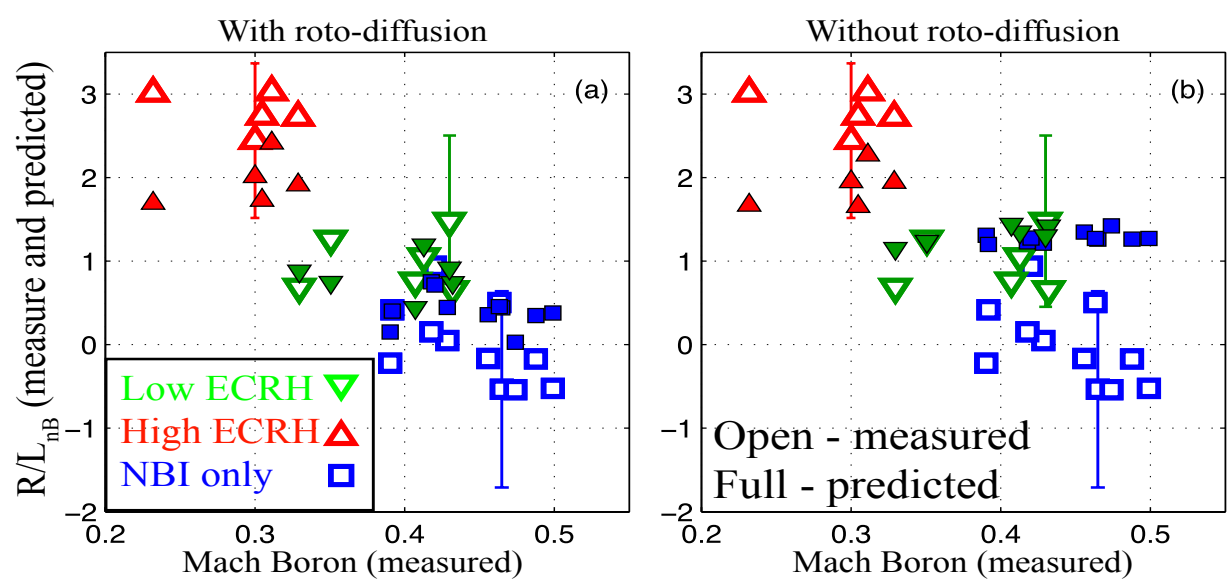

Figure 2. (a) Predicted (full symbols) and measured (open symbols) values of the normalized logarithmic boron density gradient at mid-radius as a function of the boron Mach number for the dataset considered in reference [13. Cases with only NBI heating are shown in blue squares, low ECRH power cases with green upside down triangles and high power ECRH cases in red upright triangles. In (b) the same data as in (a) is plotted, but excluding the contribution from roto-diffusion. The predicted values are the result of quasi-linear GS2 calculations.

behavior. In the high ECRH power cases, in which the rotation and rotation gradient are small, this term plays practically no role. However, in the NBI only cases, which feature high rotation velocities and strong rotation gradients, this term becomes crucial. Hence, the conclusion from reference [13], that the toroidal rotation and its radial gradient play a non-negligible role in the turbulent boron transport, remains valid. The predictions still do not reproduce entirely the hollow boron density profiles or capture the full degree of peaking in the high power ECRH cases, indications that an additional term in the turbulent flux may still be missing. However, the current description reproduces not only the trends in the experimental data, but also the absolute magnitude within the error bars of the measurement.

\section{II.3. Momentum transport}

Momentum transport analyses performed on these discharges show clearly that a simple increase in momentum diffusivity is insufficient to explain the observed flattening of the toroidal rotation [40]. Rather, the observations can only be explained by the presence of a core localized, counter-current directed, residual stress momentum flux of the same order of magnitude as the applied NBI torque. This conclusion was reached by solving the steady state toroidal angular momentum conservation equation, Eq. 1, for the unknown turbulent momentum flux using reasonable assumptions for the momentum diffusivity, $\chi_{\phi}$, and convective velocity, $V_{C}$, profiles.

$$
\Pi_{r e s}=\Pi_{N B I}+m_{i} n_{i} R\left(\chi_{\phi} \frac{\partial V_{\phi}}{\partial r}-V_{c} V_{\phi}\right)
$$



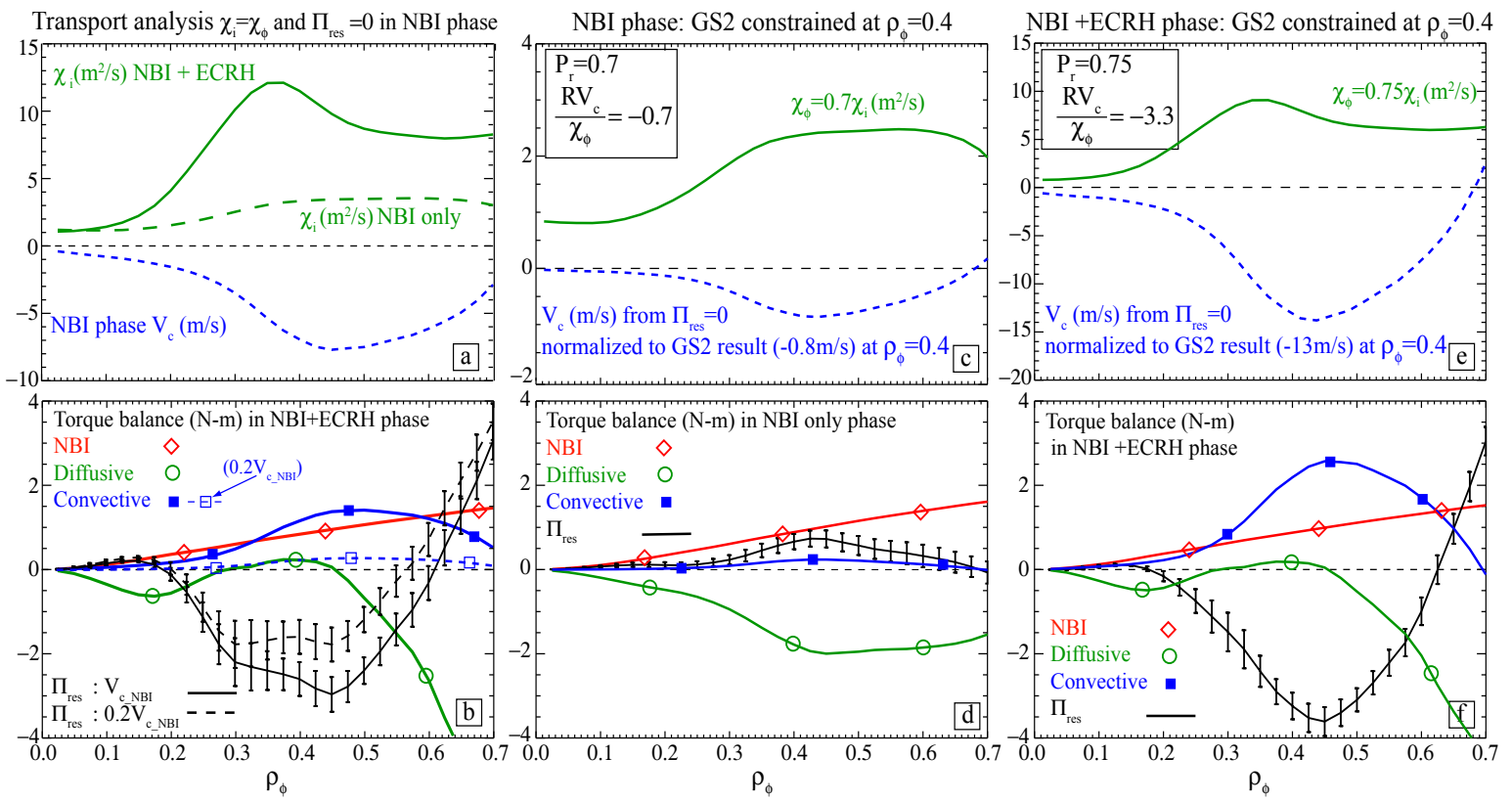

Figure 3. (a) TRANSP calculated ion thermal diffusivities for the NBI only and NBI+ECRH heated phases of discharge 26686. (b) Balance of NBI (red diamonds), diffusive (green circles), convective (blue squares), and residual stress (black solid line) torques for the NBI+ECRH phase assuming $P_{r}=1$ and making two different assumptions on the convective velocity. (c and d) Momentum transport coefficients for the NBI and NBI+ECRH phases, respectively, constrained by GS2 calculations of $P_{r}$ and $V_{C}$ at $\rho_{\phi}=0.4$. (d and f) Torque balance for NBI and NBI+ECRH phases corresponding to the transport coefficients shown in panels (c and e).

In Eq. 1. $\Pi_{r e s}$ and $\Pi_{N B I}$ are the unknown residual stress and TRANSP calculated NBI momentum fluxes, $\chi_{\phi}$ is assumed to be equal to the ion thermal diffusivity, $\chi_{i}$, and $V_{c}$ is estimated from the NBI only phase assuming $\chi_{\phi}=\chi_{i}$ and $\Pi_{r e s}=0$. The case with $\Pi_{\text {res }} \neq 0$ in the NBI phase will be considered below. Note, that the turbulent momentum flux is assumed to take the form of a residual stress torque. The measured rotation profiles could also be explained by an outward turbulent convection. However, the results presented in section III, namely, intrinsic rotation profiles with a null point $\left(V_{\phi}=0\right)$ at finite gradient, make a torque like term more probable.

The results from this analysis for AUG discharge 26686 are shown in Fig. 3. The ion thermal diffusivities for both the NBI and NBI+ECRH phases are shown in Fig. 3(a) along with the convective velocity profile obtained by assuming $\Pi_{\text {res }}=0$. In the ECRH phase there is a significant increase in $\chi_{i}$ and hence, also in $\chi_{\phi}$. However, the diffusive contribution to the momentum transport remains quite small due to the very flat rotation gradient over most of the profile, see Figs. 3(b) and 1(b). This means that in the plasma core the residual stress torque must balance against both the NBI torque and the inward convection and thus, becomes quite large. The exact magnitude and shape of the intrinsic torque depend strongly on the assumed $\chi_{\phi}$ and $V_{C}$ profiles. However, for any reasonable assumption on the Prandtl number, $P_{r}=\chi_{\phi} / \chi_{i}$, combined with an inward 
pinch of any size, a considerable intrinsic torque is still required to explain the core rotation profiles. In Fig. 3(b) the intrinsic torque has been calculated for two different assumptions on the convective velocity. First, that it remains unchanged from the NBI only phase (solid black line) and second, that the inward pinch is reduced by a factor of 5 (dashed black line). The reduced $V_{C}$ assumption illustrates that even for negligibly small convective velocities the magnitude of the counter current intrinsic torque remains of the same order as $\Pi_{N B I}$. These AUG results have parallels from other devices. Recent work from DIII-D demonstrates a strongly counter-current intrinsic momentum flux in discharges with core ECRH [29] and similar results were obtained previously on JT-60U [43].

It is also of interest to examine the momentum balance in these plasmas taking into account theoretical predictions for the momentum transport. Both heating phases of this discharge were simulated with linear runs of GS2 at $\rho_{\phi}=0.4$. These runs yield Prandtl numbers and convective velocities (Coriolis pinch [19]) of 0.7 and $-0.8 \mathrm{~m} / \mathrm{s}$ for the NBI only phase and 0.75 and $-13 \mathrm{~m} / \mathrm{s}$ for the NBI+ECRH phase. Figures 3 (c and e) show the momentum transport coefficients normalized to these values for both heating phases. In the NBI only case, the GS2 predicted inward pinch is insufficient to balance against the predicted outward diffusive term. Therefore, a co-current residual stress momentum flux is needed to conserve momentum. This is consistent with results from Alcator C-Mod and DIII-D, both of which report co-current intrinsic rotation in plasmas with $T_{i} \geq T_{e}$ [49, 50]. In the NBI+ECRH phase the predicted pinch is very large, see Fig. 3(e). This results in a co-current convective torque twice the size of the applied NBI torque, while the outward diffusive torque remains very small due to the very flat rotation gradient. Therefore, a pinch of this size would require a counter-current residual stress torque almost four times as large as the applied NBI torque to balance against all of the inward contributions, see Fig. 3(f).

\section{II.4. Momentum modulation experiments in $A U G$}

In section II.3 the intrinsic torque present in discharges heated with both NBI and ECRH power was estimated by making assumptions on the diffusive and convective portions of the momentum flux, a process which lends considerable uncertainties to the evaluation. However, through the use of modulation techniques it is possible to identify these fluxes separately. This technique has already been used on JET [31, 32, 33], DIIID [29], JT-60U [30], and NSTX [27, 28] to study momentum transport and has now been developed for AUG following the method used at JET.

The AUG momentum modulation experiments employ a $10 \mathrm{~Hz}$ on-axis NBI power modulation of $\pm 0.625 \mathrm{MW}(60 \mathrm{keV} / \mathrm{amu})$ above a baseline NBI power level of at least $3.125 \mathrm{MW}$. This results in a $\sim 5 \%$ modulation in the observed rotation, which is an ideal situation as the signal is clear, while the changes to the background heat and momentum transport are kept to a minimum. The core CXRS system was used to measure both $T_{i}$ and $V_{\phi}$ and was set to either 5 or $10 \mathrm{~ms}$ integration time: thus, providing detailed 


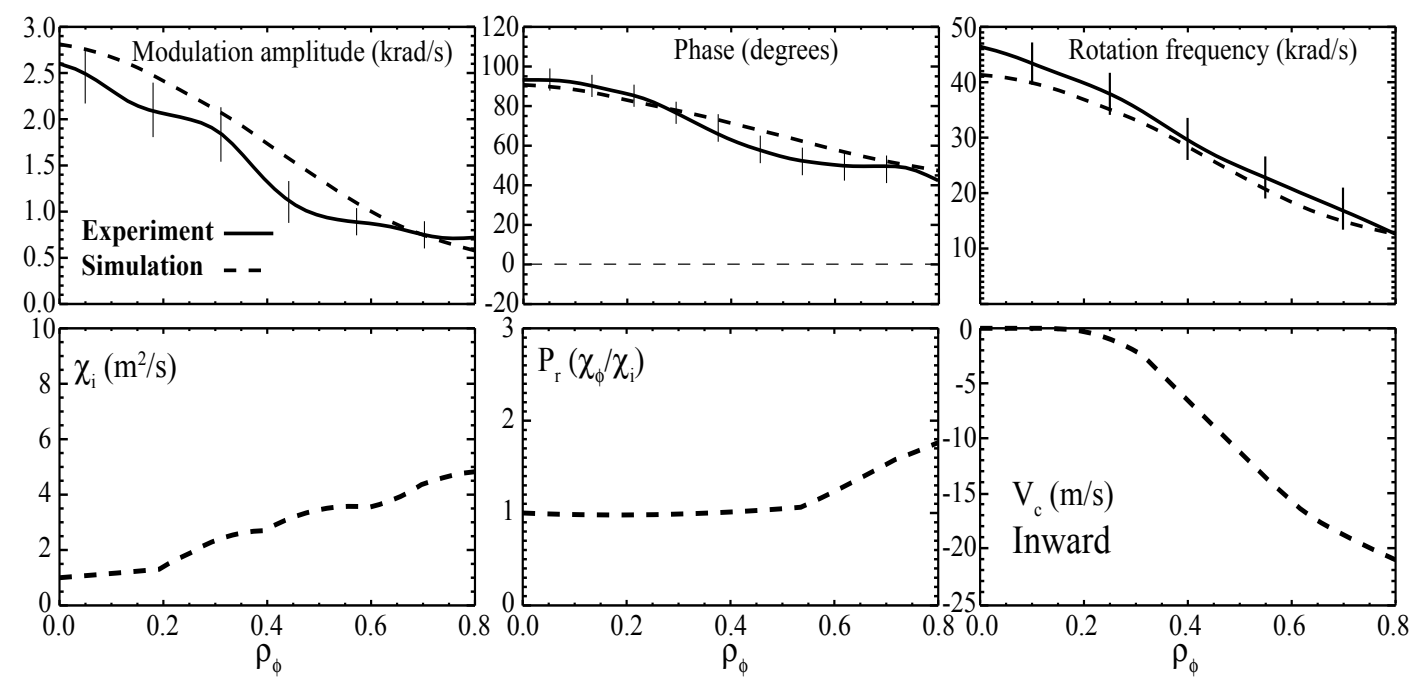

Figure 4. Top row: Experimental (solid lines) and simulated (dashed lines) rotation modulation amplitude (left), phase (middle) and steady state profile (right) for discharge 26270. Bottom row: TRANSP calculated thermal diffusivity (left), best fit Prandtl number (middle) and pinch velocity (right).

time histories of the plasma rotation response. The applied NBI torque was evaluated with TRANSP simulations which were run with $1 \mathrm{~ms}$ time resolution to capture the full dynamics of the torque, temperature, and equilibrium changes, and with 50,000 Monte Carlo particles to ensure an accurate rendering of the fast ion population and its effects on the plasma transport. The experimentally observed rotation modulation is Fourier analyzed to obtain radial phase and amplitude profiles which are simulated with the JETTO [51] code in conjunction with the steady state rotation profiles to determine the best fit $\chi_{\phi}$ and $V_{C}$ profiles. Note, that this analysis assumes a two term description of the momentum transport: a momentum transport equation of the same form as Equation 1 with $\Pi_{\text {res }}=0$. Therefore, care must be taken in the interpretation of results if the intrinsic torques are not negligible compared to the externally applied torque.

The analysis of these experiments is ongoing and will be the subject of an upcoming publication. However, the preliminary results yield some important information and demonstrate the feasibility of the modulation technique on AUG. The top row of Fig. 4 shows a comparison of the experimental and simulated rotation modulation amplitude, phase, and steady state rotation profile for an H-mode plasma heated with $0.8 \mathrm{MW}$ of ECRH and 2.5 MW of NBI power in addition to the $0.625 \mathrm{MW}$ from the modulation. The steady state profile is well reproduced over the entire profile as is the phase, while the modulation amplitude tends to be a little high. Still, it is reproduced within $20 \%$ of the experimental data. The best fit transport coefficients are shown in the bottom row of Fig. 4. Here, one can see that the Prandtl number is found to be close to one, consistent with the strong coupling between $\chi_{i}$ and $\chi_{\phi}$ expected with ITG turbulence. The best fit convective velocity shows an inward pinch that is similar to the profile estimated in section 1, see Fig. 3. These results are consistent with those from other 
devices and also with the assumptions on $P_{r}$ and $V_{C}$ made in section II.3. However, one should keep in mind that these results were obtained with the assumption $\Pi_{r e s}=0$. If in fact a co-current (counter-current) intrinsic momentum flux was present in this plasma then this would lead to an under (over) estimation of the determined $P_{r}$ and $V_{C}$ profiles.

\section{Intrinsic toroidal rotation in ASDEX Upgrade}

\section{III.1. Intrinsic rotation measurements using short NBI blips}

On AUG all CXRS measurements are made using one of the neutral heating beams. Unfortunately, all of the heating beams impart significant momentum to the plasma and typically provide sufficient heating power to initiate an $\mathrm{L}-\mathrm{H}$ transition. These circumstances have traditionally limited momentum transport studies on AUG to NBI heated H-mode scenarios. However, the upgraded core CXRS system can now operate with $4 \mathrm{~ms}$ integration time, which enables the use of $12-16 \mathrm{~ms}$ NBI blips to make rotation measurements while minimizing the amount of applied torque and avoiding an L-H transition for most plasma conditions.

The short NBI blip scenario is not entirely torque free, as can be seen by observing the temporal evolution of the toroidal rotation during the blip. However, often very little to no evolution is observed in the first 8-12 ms, making these measured profiles representative of the true intrinsic rotation. In addition, when significant evolution is observed the rotation can be extrapolated backward in time to determine the true intrinsic rotation, a technique that has been employed previously on other tokamak devices [52]. An example of AUG beam blip analysis for an ohmic L-mode plasma, which demonstrates the maximum level of evolution observed to date, is shown in Fig. 5. The measured rotation profiles are shown on the left, while the plots on the right illustrate the temporal evolution for a few individual radial points and the linear backward extrapolation used to determine the true intrinsic rotation. Here, the backward extrapolation lowers the rotation profile by $2-3 \mathrm{~km} / \mathrm{s}$ relative to the first measured profile and $5 \mathrm{~km} / \mathrm{s}$ when compared to the average, but the shape of the profile is almost unchanged.

\section{III.2. AUG intrinsic rotation database}

Using the beam blip technique, an intrinsic rotation profile database has been developed at AUG containing points from ohmic and ECRH L-modes as well as ECRH and ICRH $\mathrm{H}$-modes. All of the profiles in this database are from diverted, single null plasmas with the $\mathrm{X}$-point in the favorable ion drift direction and similar elongation $(1.6<\kappa<1.8)$ and averaged triangularity $(0.15<\delta<0.27)$. The database includes a plasma current variation between 0.6 and 1.0 MA, magnetic fields between -1.5 and $-2.7 \mathrm{~T}, q_{95}$ values between 3 and 8, and line averaged core electron densities between 1 and $8.5 \mathrm{e}^{19} \mathrm{~m}^{-3}$. There is also a significant variation in the core electron temperature $(0.9-10.5 \mathrm{keV})$, 

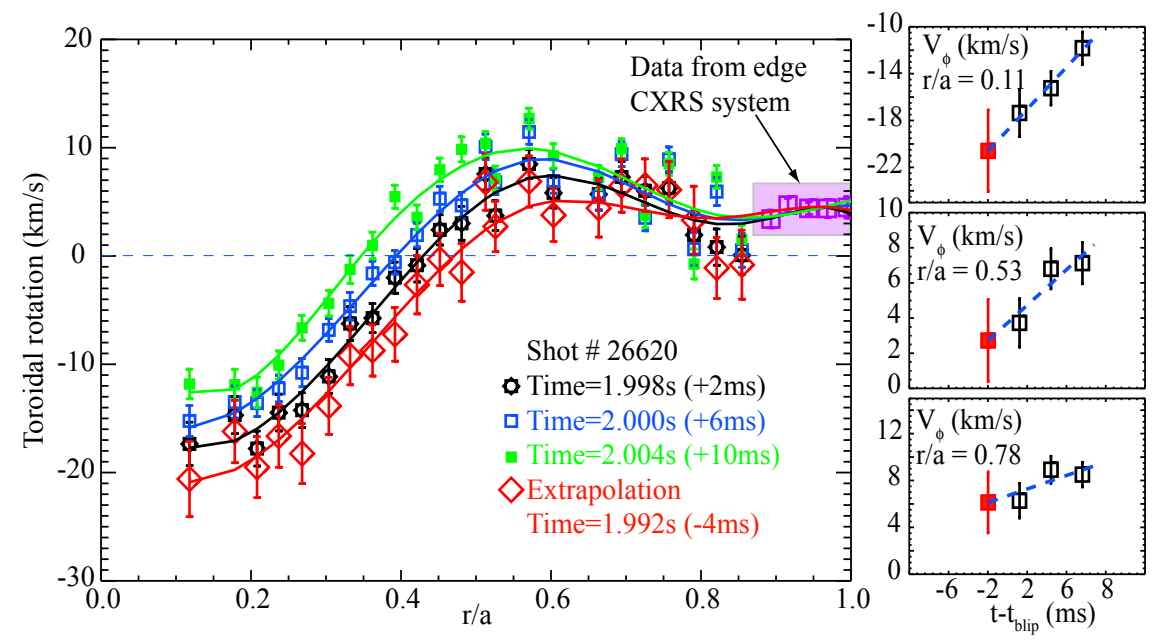

Figure 5. Toroidal rotation profiles from $2 \mathrm{~ms}$ (black), $6 \mathrm{~ms}$ (blue), and $10 \mathrm{~ms}$ (green) into an NBI blip as well as the backward extrapolation to the rotation profile $2 \mathrm{~ms}$ before (red). The highlighted purple points are from the edge CXRS diagnostic, and have been averaged over the NBI blip. On the right hand side the temporal evolution of three individual radial points is shown along with the linear fit used to determine the intrinsic rotation.

while the core ion temperature is fairly constant only varying between 0.7 and $1.6 \mathrm{keV}$. This is because all of the relevant heating scenarios (ohmic, ECRH and ICRH) produce dominant electron heating in AUG.

The profiles in the database are the measured charge exchange low field side impurity profiles, typically either carbon or boron. However, the main ion rotation has been calculated for the entire database using a neoclassical correction to the impurities and is found to be within $3 \%$ of the impurity rotation for most points. Therefore, the impurity rotation is taken as representative of the bulk behavior. The selection criteria to include points in the database are very general. They must be characterized by good quality CXRS beam blip data, good $n_{e}$ and $T_{e}$ profiles, and come from a steady-state period of a plasma discharge without external momentum input and without significant MHD mode activity. Many of the discharges do display sawtooth activity and care has been taken to exclude the sawtooth affected portion of the rotation profiles from the database. Practically, this means that many of the rotation profiles, particularly those at higher currents, only extend inward to $\rho_{\phi} \simeq 0.25$.

Within the database the edge toroidal rotation is almost always in the co-current direction with a flat to co-current gradient (rotation increasing in the co-current direction with decreasing radius) regardless of plasma parameters, while the core rotation shows considerable variability in both sign and gradient. Many of the intrinsic toroidal rotation profiles, such as the one shown in Fig. 5, switch direction from counter-current to cocurrent as a function of radius. This behavior, having a null point in the profile at finite rotation gradient, is clear evidence that the mechanism at work is in fact a residual stress torque and not a convection. This is certainly true for these intrinsic rotation 
cases and it is also likely true for the NBI+ECRH H-modes presented in section II.3 although this can not be stated definitively.

As a starting point we examine the thermal Mach number $\left(M=V_{\phi} / V_{t h}\right)$ at $\rho_{\phi}=0.4$ for the entire database as a function of local effective collisionality $\left(\nu=\nu_{e i} /\left(C_{S} / R\right)\right)$, see Fig. 6(a). $\nu_{e i}$ is the electron ion collision frequency and $C_{S}$ is the ion sound speed. Here, the database has been divided into two subsets based on the condition $T_{e} / T_{i}=2$, which effectively constitutes a separation into low (red full symbols) and high (blue open symbols) collisionality points. This was done because these two subsets appear to display distinctly different rotation behaviors. The low collisionality points correspond to $T_{e} / T_{i}$ ratios between 2 and 6 , and typically quite low plasma densities and electron density gradients scale lengths $\left(R / L_{n e}\right)$. Under these conditions the core toroidal rotation is always co-current with a co-current gradient. The high collisionality subset, however, displays considerable variation in both sign and gradient. As such, it presents the more interesting and fruitful data set to understand the rotation behavior as a function of plasma parameters.

In these plasmas there are no externally applied torques so the momentum transport becomes a balance between the diffusive, convective and residual stress momentum fluxes: $\Pi_{r e s}+n_{i} m_{i} R\left(\chi_{\phi} d V_{\phi} / d r-V_{c} V_{\phi}\right)=0$. If the convective contribution is small then the gradient of the rotation is directly proportional to the residual stress. Given this, the database is examined with respect to the plasma turbulence as well as to the plasma parameters known to influence the type of turbulence, such as $\nabla T_{e}, \nabla T_{i}$ and $\nabla n_{e}$. In Fig. 6 (b) the rotation gradient normalized to the thermal velocity, $U^{\prime}=\left(R / V_{t h}\right)\left(d V_{\phi} / d r\right)$, at $\rho_{\phi}=0.4$ is shown as a function of the frequency of the dominant turbulent mode, $\omega_{r}$, as calculated by GS2. Here, despite the scatter, one can see a linear dependence of $U^{\prime}$ on $\omega_{r}$ for the high collisionality points with co-current gradients occurring for ITG plasmas and increasingly counter-current gradients for TEM. The low collisionality points show no dependence on $\omega_{r}$. An examination of the rotation gradients as a function of $\nabla T_{e}$ and $\nabla T_{i}$ shows little to no correlation. However, a clear relationship is seen with the electron density gradient (Fig. 6(c)), which even incorporates the low collisionality subset. For moderate temperature gradients, a steep density gradient can destabilize TEM turbulence leading to negative rotation gradients, while for low density gradients the plasma remains in ITG. In these situations, the plasma turbulence is very sensitive to small changes in the $\nabla n_{e}$ potentially helping to explain the high sensitivities to electron density seen on multiple devices.

The observed correlations with the dominant turbulent mode and with the electron density gradient are reminiscent of the type of residual stress momentum flux suggested in reference [53] and considered in detail by Camenen et al. [21]. This work considers the residual stress momentum flux produced by the poloidal tilt of turbulent eddies due to profile shearing. It finds a co-current momentum flux for ITG turbulence and a counter-current flux for TEM, with the magnitude of the residual stress proportional to the electron density gradient. These predictions are qualitatively consistent with the initial results from the AUG intrinsic rotation database. Note that in Fig. 6, many 

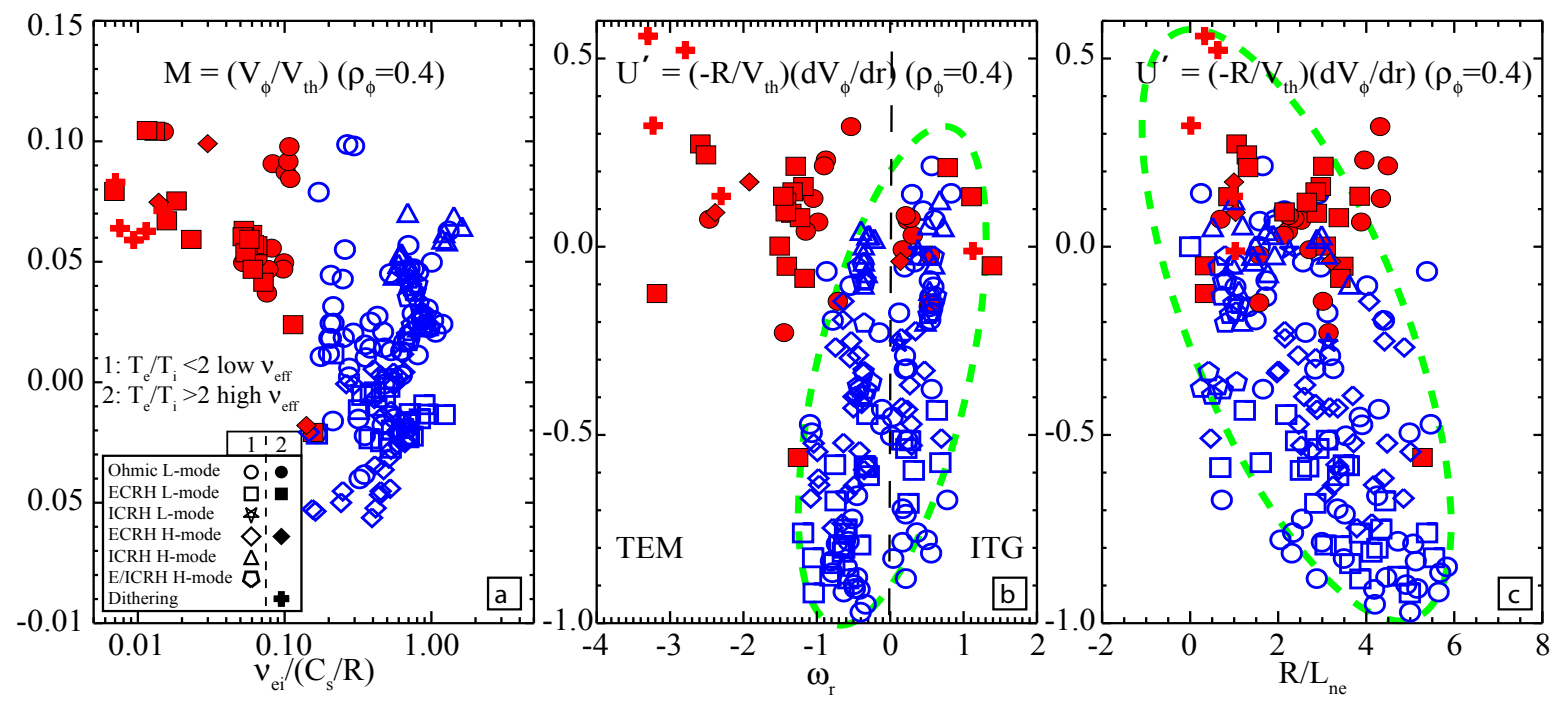

Figure 6. (a) Thermal Mach number versus local plasma collisionality, (b) normalized toroidal rotation gradient, U', versus the frequency of the dominant turbulent mode, and (c) U' versus the normalized electron density gradient. Red points (full) correspond to $T_{e} / T_{i}>2$ (low collisionality) and blue points (open) to $T_{e} / T_{i}<2$ (high collisionality).

of the lowest collisionality points are also the deepest in the TEM regime (red crosses and squares) and correspond to very low values of $R / L_{n e}$. It is possible that these plasmas, despite being TEM dominated, exist in a corner of parameter space for which the turbulent momentum flux is small due to the very flat density gradients. Given this picture, strongly TEM dominated plasmas, which are well past the maximum in the turbulent peaking of the electron density (see section II.2), are not likely to exhibit counter-current core rotation, but rather are strongly influenced by the sign and gradient of the edge rotation. This idea would bring the high and low collisionality subsets together into a consistent description of the core intrinsic rotation. More quantitative comparisons are the topic of ongoing work.

In the work presented here no differentiation was made with respect to heating method or confinement regime. Thus, it appears that the core rotation in ECRH and ICRH H-modes are controlled by the same turbulent mechanisms that determine L-mode rotation. Given this, whether the core rotation in ICRH heated H-modes spins up in the co-current direction as typically observed on Alcator C-Mod [54] or remains hollow and in the counter current direction as recently observed on JET [55], may be entirely consistent, and explained by the local plasma parameters and turbulent transport.

\section{Summary and Conclusions}

Core momentum and particle transport in AUG have been examined in a wide variety of plasma discharges and via several different methods. Experiments were performed in which ECRH power was added to NBI heated H-modes causing the electron and 
impurity ion density profiles to peak and the core toroidal rotation to flatten. Turbulence calculations of these plasmas show a change in the dominant regime from ITG to TEM due to the ECRH induced changes in the electron and ion temperature profiles. The impurity and electron density behaviors are fully explained by the changes in the turbulent transport and are well reproduced by simulations. Momentum transport analyses of the high power ECRH cases (TEM) demonstrate the presence of a core localized, counter-current directed, residual stress momentum flux of the same order of magnitude as the applied NBI torque. These analyses relied on the assumption that $P_{r} \simeq 1$ an assumption that is supported by the results from the first momentum modulation experiments performed on AUG.

The newly upgraded core CXRS system has enabled the first measurements of intrinsic rotation to be made on AUG through the use of 12-16 ms NBI blips. Using this technique an intrinsic toroidal rotation database has been developed, which can be used to test dependencies of theoretically predicted residual stress momentum fluxes. The database shows correlations between the gradient of the toroidal rotation and both the frequency of the dominant turbulent mode and $R / L_{n e}$, qualitatively consistent with the theoretically predicted turbulent momentum fluxes due to tilted turbulent eddies [21. The database includes points from ohmic, ECRH and ICRH L-modes as well as ECRH and ICRH H-modes. The similarities in the rotation behavior as a function of local plasma parameters in these discharges suggest that the same turbulent mechanisms govern the toroidal rotation regardless of plasma type or heating method.

The results from the intrinsic rotation database are consistent with those obtained in NBI H-modes with high levels of ECRH power in that both sets of experiments demonstrate the presence of a counter-current directed residual stress momentum flux when the plasma is dominated by TEM turbulence. If turbulent momentum fluxes due to tilted eddies is in fact the dominant mechanism determining core intrinsic rotation then there would also necessarily be a co-current intrinsic momentum flux present in ITG dominated plasmas. This possibility is not taken into account in the analysis of modulation experiments like those presented in this work, which assume a two term description of the momentum transport. This would lead to an underestimate of the transport coefficients from this type of analysis and should be kept in mind when interpreting these results.

\section{Acknowledgments}

The authors would like to acknowledge the efforts and contributions of the entire ASDEX Upgrade team and in particular the NBI and ECH groups. This work was in part supported by the European Communities under the contract of Association between EURATOM/IPP and carried out within the framework of the EFDA Workprogramme 2011 Transport Task Agreement WP11-TRA-04. The views and opinions expressed herein do not necessarily reflect those of the European Commission. 
[1] H. Biglari, P. H. Diamond, P. W. Terry. Phys. Fluids B, 2:1, 1990.

[2] T. S. Hahm. Phys. Plasmas, 1:2940, 1994.

[3] T. S. Hahm and K. H. Burrell. Phys. of Plasmas, 2:1648, 1995.

[4] K. H. Burrell. Phys. Plasmas, 4:1499, 1997.

[5] P. W. Terry. Rev. Mod. Phys., 72:109, 2000.

[6] E. J. Strait et al. Phys. Rev. Lett, 74:2483, 1995.

[7] A. M. Garofalo et al. Phys. Rev. Lett., 89:235001, 2002.

[8] H. Reimerdes et al. Phys. Rev. Lett., 98:055001, 2007.

[9] R. J. Buttery et al. 22nd IAEA Fusion Energy Conference, Geneva Switzerland, 2008.

[10] P. A. Politzer et al. Nucl. Fusion, 48:075001, 2008.

[11] D. J. Ward et al. Fusion Eng. Design, 75-79:1221, 2005.

[12] C. Angioni et al. Plasma Phys. Control. Fusion, 51:124017, 2009.

[13] C. Angioni et al. Nucl. Fusion, 51:023006, 2011.

[14] M. Maslov et al. Nucl. Fusion, 49:075037, 2009.

[15] D. Ernst et al. Phys. Plasmas, 11:2637, 2004.

[16] E. Fable et al. Plasma Phys. Control. Fusion, 52:015007, 2010.

[17] N. Mattor and P.H. Diamond. Phys. Fluids, 31:1180, 1988.

[18] A. G. Peeters et al. Plasma Phys. Control. Fusion, 48:B413, 2006.

[19] A. G. Peeters, C. Angioni and D. Strintzi. Phys. Rev. Lett., 98:265003, 2007.

[20] Y. Camenen et al. Phys. Rev. Lett., 102:125001, 2009.

[21] Y. Camenen et al. Nucl. Fusion, 51:073039, 2011.

[22] W. X. Wang et al. Nucl. Fusion, 18:042502, 2011.

[23] O. D. Gurcan et al. Phys. Plasmas, 17:032509, 2010.

[24] E. S. Yoon and T. S. Hahm. Nucl. Fusion, 50:064006, 2010.

[25] C. J. McDevitt et al. Phys. Plasmas, 16:052302, 2009.

[26] P. H. Diamond et al. Nucl. Fusion, 49:045002, 2009.

[27] W. M. Solomon et al. Phys. Rev. Lett., 101:065004, 2008.

[28] S. M. Kaye et al. Nucl. Fusion, 49:045010, 2009.

[29] W. M. Solomon et al. Phys. Plasmas, 17:056108, 2010.

[30] M. Yoshida et al. Nucl. Fusion, 47:856, 2007.

[31] T. Tala et al. Phys. Rev. Lett, 102:075001, 2009.

[32] G. Tardini et al. Nucl. Fusion, 49:085010, 2009.

[33] P. Mantica et al. Phys. Plasmas, 17:092505, 2010.

[34] Y. Camenen et al. Phys. Rev. Lett., 105:135003, 2010.

[35] J. E. Rice et al. Phys. Rev. Lett., 106:215001, 2011.

[36] B. P. Duval et al. Plasma Phys. Control. Fusion, 49:B195, 2007.

[37] A. Scarabosio et al. Plasma Phys. Control. Fusion, 48:663, 2006.

[38] J. E. Rice et al. Plasma Phys.Control. Fusion, 50:124042, 2008.

[39] J. E. Rice et al. Nucl. Fusion, 51:083005, 2011.

[40] R. M. McDermott et al. Plasma Phys. Control. Fusion, 53:035007, 2011.

[41] J. S. deGrassie et al. Phys. Plasmas, 14:056115, 2007.

[42] W. M. Solomon et al. Nucl. Fusion, 51:073010, 2011.

[43] M. Yoshida et al. Phys. Rev. Lett., 103:065003, 2009.

[44] W. Dorland et al. Phys. Rev. Lett, 85:5579, 2000.

[45] N. Kluy et al. Phys. Plasmas, 12:122302, 2009.

[46] C. Angioni et al. Phys. Plasmas, 12:040701, 2005.

[47] J. Stober et al. Nucl. Fusion, 43:1265, 2003.

[48] Y. Camenen et al. Phys. Plasmas, 16:012503, 2009.

[49] W. M. Solomon et al. Plasma Phys. Control. Fusion, 49:B313, 2007.

[50] J. E. Rice et al. Nucl. Fusion, 39:1175, 1999.

[51] G. Genacchi, et al. JETTO: A free boundary plasma transport code (basic version) Rapporto 
ENEA RT/TIB 1988(5), 1988.

[52] J. S. deGrassie et al. Phys. Plasmas, 11:4323, 2004.

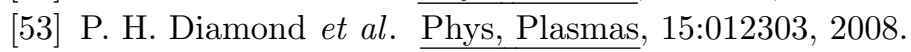

[54] J. E. Rice et al. Nucl. Fusion, 47:1618, 2007.

[55] M.F.F. Nave et al. Phys. Rev. Lett., 105:105005, 2010. 